\title{
A tool to analyze robust stability for constrained nonlinear MPC
}

\author{
Lino O. Santos ${ }^{\mathrm{a}, *}$, Lorenz T. Biegler ${ }^{\mathrm{b}}$, José A.A.M. Castro ${ }^{\mathrm{a}, \mathrm{A}}$ \\ ${ }^{a}$ GEPSI - PSE Group, Department of Chemical Engineering, University of Coimbra - Pólo II, 3030-790 Coimbra, Portugal \\ ${ }^{\mathrm{b}}$ Department of Chemical Engineering, Carnegie Mellon University, Pittsburgh, PA 15213, USA
}

Received 15 August 2007; accepted 3 October 2007

\begin{abstract}
A sufficient condition for robust asymptotic stability of nonlinear constrained model predictive control (MPC) is derived with respect to plant/model mismatch. This work is an extension of a previous study on the unconstrained nonlinear MPC problem, and is based on nonlinear programming sensitivity concepts. It addresses the discrete time state feedback problem with all states measured. A strategy to estimate bounds on the plant/model mismatch is proposed that can be used off-line as a tool to assess the extent of model mismatch that can be tolerated to guarantee robust stability.
\end{abstract}

(c) 2007 Elsevier Ltd. All rights reserved.

Keywords: Nonlinear model predictive control; Robust stability; Sensitivity analysis

\section{Introduction}

A prominent research topic for nonlinear model predictive control (MPC) is the development of a theoretical framework for stability of the closed-loop system in the presence of disturbances and modeling errors. A broad review by Mayne et al. [7] on constrained MPC points out that while research on stability has reached a relatively mature stage, further research is required to develop implementable robust MPC for nonlinear systems. More recent studies $[6,4,5]$ have analyzed the robust design of nonlinear MPC discrete time controllers. There a robustness margin is derived in conjunction with the Lyapunov stability conditions. A related analysis is also presented for unconstrained nonlinear MPC problems in [10]. On the other hand, the treatment of constrained MPC systems is more difficult, and in $[6,5]$ a robust analysis is presented based on Pontryagin difference sets, which can lead to conservative controllers.

\footnotetext{
* Corresponding author. Tel.: +351 239798700; fax: +351 239798703.

E-mail addresses: lino@eq.uc.pt (L.O. Santos), biegler@cmu.edu (L.T. Biegler).

Deceased.
}

In this study, we consider a different approach based on exact penalty functions. Here, we develop a framework that can be used to evaluate off-line, the closed-loop robustness of a constrained MPC system in the presence of plant/model mismatch. This is a direct extension of previous work on the unconstrained case [10] for the discrete state feedback problem. Both the plant and model are described using nonlinear state-space models. The results in this paper lead to a criterion that is a sufficient condition for asymptotic stability in the presence of plant/model mismatch. We note, however, that we do not treat the weaker (but very useful) property of input to state stability (ISS), covered in [6,5], although in future work we plan to extend this framework to deal with ISS as well.

The paper is organized as follows. Section 2 is devoted to preliminary definitions and assumptions on the modeling errors, and to a brief description of the MPC problem. In Section 3, we analyze both the perfect and model mismatch MPC cases, by exploiting the properties of the exact penalty function, and we establish a sufficient condition for robust asymptotic stability. In Section 4, using nonlinear programming sensitivity concepts, we characterize this sufficient condition for the MPC problem with a general cost function. We further detail this characterization for the 
case of a quadratic cost function, and we obtain a quantitative, computable bound on the plant/model uncertainty. This bound can be estimated through off-line calculations using a procedure that constitutes a tool to analyze robust stability for constrained MPC. These results are illustrated in Section 5 with a simple example. Finally, concluding remarks regarding the analysis of conditions for robust stability of MPC in the presence of plant/model mismatch are given in Section 6.

\section{Definitions and notation}

For this study we consider only the state feedback case and assume that at every time index $i$ all the states can be measured accurately. We assume the state dynamics of the plant are described by the following nonlinear, continuous-time set of equations:

$\dot{x}^{\mathrm{p}}=\boldsymbol{f}^{\mathrm{p}}\left(x^{\mathrm{p}}, u\right)$,

where $x^{\mathrm{p}} \in \mathbb{R}^{n_{\mathrm{s}}}$ is the vector of states and $u \in \mathbb{R}^{n_{\mathrm{m}}}$ is the vector of inputs, with $\boldsymbol{f}^{\mathrm{p}}: \mathbb{R}^{n_{\mathrm{s}}} \times \mathbb{R}^{n_{\mathrm{m}}} \rightarrow \mathbb{R}^{n_{\mathrm{s}}}$.

The stationary discrete-time plant counterpart of (1) is

$x_{k+1}^{\mathrm{p}}=f^{\mathrm{p}}\left(\Delta t ; x_{k}^{\mathrm{p}}, u_{k}\right)$,

where $\Delta t$ is the sampling period and $f^{\mathrm{p}}: \mathbb{R}^{n_{\mathrm{s}}} \times \mathbb{R}^{n_{\mathrm{m}}} \rightarrow \mathbb{R}^{n_{\mathrm{s}}}$. We will drop the $\Delta t$ for convenience. A model with the same dimension as (2) is considered for the MPC framework:

$x_{k+1}=f\left(x_{k}, u_{k}\right)$,

where $x_{k} \in \mathbb{R}^{n_{s}}$ is the vector of nominal states, $u_{k}$ is the same vector of inputs as in (2), with $f: \mathbb{R}^{n_{\mathrm{s}}} \times \mathbb{R}^{n_{\mathrm{m}}} \rightarrow \mathbb{R}^{n_{\mathrm{s}}}$. We consider $\left(x_{k}^{\mathrm{p}}, u_{k}\right)=\left(x_{k}, u_{k}\right)=(0,0)$ the point at which both the plant and the model operate at steady state, such that $f(0,0)=f^{\mathrm{p}}(0,0)=0$.

As in Keerthi and Gilbert [3] we apply the definition of a function belonging to class $\mathscr{K}_{\infty}$. Here, a function $W(r): \mathbb{R}_{+} \rightarrow \mathbb{R}_{+}, r \in \mathbb{R}_{+}$, belongs to class $\mathscr{K}_{\infty}$ if: (a) it is continuous; (b) $W(r)=0 \Longleftrightarrow r=0$; (c) it is nondecreasing; (d) $W(r) \rightarrow \infty$ when $r \rightarrow \infty$. We define $\|\cdot\|$ as the Euclidean norm and assume there exists a modeling bound function $W_{\mathrm{m}}(\cdot) \in \mathscr{K}_{\infty}$ such that

$\left\|f^{\mathrm{p}}\left(x_{k}^{\mathrm{p}}, u_{k}\right)-f\left(x_{k}, u_{k}\right)\right\| \leqslant W_{\mathrm{m}}\left(\left\|x_{k}\right\|\right)$,

and positive constants $K_{\mathrm{m}}$ and $\gamma$ such that

$W_{\mathrm{m}}\left(\left\|x_{k}\right\|\right)=K_{\mathrm{m}}\left\|x_{k}\right\|^{\gamma}$.

The MPC problem minimizes

$\Psi\left(x_{i}, \boldsymbol{s}_{i}\right)=\sum_{k=i}^{i+p-1} h\left(x_{k}, u_{k}\right)+h_{\mathrm{F}}\left(x_{i+p}\right)$,

where $\Psi: \mathbb{R}^{n_{\mathrm{s}}} \times \mathbb{R}^{n_{\mathrm{m}}} \rightarrow \mathbb{R}, \Psi(0,0)=0$. Here $h(x, u) \in \mathscr{K}_{\infty}$ and $h_{\mathrm{F}}(x) \in \mathscr{K}_{\infty}$ are general cost functions, $x_{i}$ is the initial state vector at the time index $i, i \geqslant 0$, and $s_{i}$ is the solution vector over the predictive horizon, given by

$$
\begin{aligned}
& \boldsymbol{s}_{i}^{\mathrm{T}}=\left[\begin{array}{llll}
s_{i}^{\mathrm{T}} & s_{i+1}^{\mathrm{T}} & \cdots & s_{i+p}^{\mathrm{T}}
\end{array}\right], \quad \text { where } s_{i+k}^{\mathrm{T}}=\left[\begin{array}{ll}
x_{i+k}^{\mathrm{T}} & u_{i+k}^{\mathrm{T}}
\end{array}\right], \\
& \quad k=0,1, \ldots, p .
\end{aligned}
$$

This formulation allows a shorter input horizon $m$, with $m \leqslant p$ and $u_{k}=u_{i+m-1}, k=i+m, \ldots, i+p$. Traditionally, the decision variables of the MPC problem are the control profiles. In the optimization framework used in this study the state profiles are decision variables as well. It uses a multiple shooting method to solve (3) over the predictive horizon $[11,9]$. State and control constraints over this horizon are included in the MPC formulation, set as lower and upper bounds - subscripts $\mathrm{L}$ and $\mathrm{U}$,

$b_{x}\left(x_{k}\right)=\left[\begin{array}{c}x_{k}-x_{\mathrm{U}} k \\ -x_{k}+x_{\mathrm{L}} k\end{array}\right] \leqslant 0$ and

$b_{u}\left(u_{j}\right)=\left[\begin{array}{c}u_{j}-u_{\mathrm{U}} j \\ -u_{j}+u_{\mathrm{L}} j\end{array}\right] \leqslant 0$

with $k=i+1, \ldots, i+p$, and $j=i, \ldots, i+m-1$. We define the vector of inequality constraints of the problem at $i$ as

$\boldsymbol{b}\left(\boldsymbol{s}_{i}\right)^{\mathrm{T}}=\left[b_{x}\left(x_{i+1}\right)^{\mathrm{T}} \cdots b_{x}\left(x_{i+p-1}\right)^{\mathrm{T}} \quad b_{u}\left(u_{i}\right)^{\mathrm{T}} \cdots b_{u}\left(u_{i+m-1}\right)^{\mathrm{T}}\right]$.

Finally, within this framework we impose two types of terminal constraints to enforce the nominal stability of the finite horizon MPC controller. The most straightforward is a zero state constraint with $x_{i+p}=0$, although this constraint may become difficult to enforce. In addition, we consider the dual mode controller. Here, $h_{\mathrm{F}}(\cdot)$ is chosen as an upper bound on the cost of a stabilizing controller, say $u=\kappa(x)$, that regulates the system, whenever it is within the neighborhood of the origin defined by $\|x\| \leqslant \delta$. This controller should also be feasible to bounds on $u$. Nominal stability then follows by applying the condition $[7,5]$

$h_{\mathrm{F}}(f(x, \kappa(x)))-h_{\mathrm{F}}(x) \leqslant-h(x, \kappa(x)) \quad \forall x \mid\|x\| \leqslant \delta$.

We denote by $\mathscr{P}\left(x_{i}\right)$ the MPC problem solved at every time index $i, i \geqslant 0$, given by

$$
\begin{array}{cl}
\min _{\boldsymbol{s}_{i}} & \Psi\left(x_{i}, \boldsymbol{s}_{i}\right) \\
\text { s.t. } & \boldsymbol{c}\left(x_{i}, \boldsymbol{s}_{i}\right)=0, \\
& \boldsymbol{b}\left(\boldsymbol{s}_{i}\right) \leqslant 0,
\end{array}
$$

where

$$
\boldsymbol{c}\left(x_{i}, \boldsymbol{s}_{i}\right)=\left[\begin{array}{c}
x_{i+1}-f\left(x_{i}, u_{i}\right) \\
\vdots \\
x_{i+p}-f\left(x_{i+p-1}, u_{i+p-1}\right) \\
x_{i+p}
\end{array}\right]
$$

for the zero state constraint or

$$
\boldsymbol{c}\left(x_{i}, \boldsymbol{s}_{i}\right)=\left[\begin{array}{c}
x_{i+1}-f\left(x_{i}, u_{i}\right) \\
\vdots \\
x_{i+p}-f\left(x_{i+p-1}, u_{i+p-1}\right) \\
\left\|x_{i+p}\right\|-\delta+\sigma^{2}
\end{array}\right]
$$


for the dual mode controller, with $\sigma$ as a slack variable. Optional constraints can also be added for a shorter input horizon, $m \leqslant p$. We assume in this analysis that $\boldsymbol{s}_{i}$ is also a feasible solution for (11)-(13) and that there exists a sufficiently long horizon that ensures an admissible trajectory to satisfy the terminal constraints and (13).

\section{Stability analysis}

To extend the analysis made for the unconstrained case by Santos and Biegler [10] to (11)-(13) we use an exact penalty formulation as developed by Oliveira and Biegler [8]. This approach converts (11)-(13) to the problem $\mathscr{P}_{\rho}\left(x_{i}\right)$ :

$$
\begin{array}{ll}
\min _{\boldsymbol{s}_{i}} & \Upsilon\left(x_{i}, \boldsymbol{s}_{i}, \rho_{i}\right) \\
\text { s.t. } \quad \boldsymbol{c}\left(x_{i}, \boldsymbol{s}_{i}\right)=0, \\
\text { with } \quad \Upsilon\left(x_{i}, \boldsymbol{s}_{i}, \rho_{i}\right)=\Psi\left(x_{i}, \boldsymbol{s}_{i}\right)+P\left(\boldsymbol{s}_{i}, \rho_{i}\right), \\
\Upsilon: \mathbb{R}^{n_{\mathrm{s}}} \times \mathbb{R}^{n_{\mathrm{m}}} \rightarrow \mathbb{R}, \Upsilon(0,0,0)=0, \text { where } \\
P\left(\boldsymbol{s}_{i}, \rho_{i}\right) \\
\quad=\rho_{i} \cdot\left[\sum_{k=i+1}^{i+p-1} \max \left\{0, b_{x}\left(x_{k}\right)\right\}+\sum_{k=i}^{i+m-1} \max \left\{0, b_{u}\left(u_{k}\right)\right\}\right],
\end{array}
$$

and $\rho_{i}$ is the penalty parameter. We remark that $P\left(s_{i}, \rho_{i}\right)$ is bounded from below by zero as well. Use of the exact penalty function is motivated by the property that the solutions to $\mathscr{P}\left(x_{i}\right)$ and $\mathscr{P}_{\rho}\left(x_{i}\right)$ are equivalent for all $\rho_{i}>\left\|\omega_{i}^{*}\right\|_{\infty}$, where $\omega_{i}^{*}$ is the vector of the Lagrange multipliers associated to $\mathscr{P}\left(x_{i}\right)$ [2]. The condition on $\rho_{i}$ ensures that the control and state profiles do not exceed the region delimited by (8) over $p$. We will assume that the parameter $\rho_{i}$ can be chosen in advance to be sufficiently large, i.e., $\rho \geqslant \max _{i}\left\{\rho_{i}\right\}$, and that if $\rho_{i}$ cannot be bounded, then $\mathscr{P}\left(x_{i}\right)$ has no feasible solution. Of course, feasible solutions of $\mathscr{P}\left(x_{i}\right)$ cannot always be guaranteed and for this reason, a 'reasonable' value can be chosen for $\rho$ so that solutions of $\mathscr{P}_{\rho}\left(x_{i}\right)$ can be considered even if they cannot always satisfy the bound constraints. To simplify the notation we set

$\Upsilon^{*}\left(x_{i}\right)=\Upsilon\left(x_{i}, s_{i}^{*}, \rho\right)$.

For our analysis we also assume the equality constraints (15) can be satisfied for all cases of model mismatch. Usually, this can be satisfied by choosing a sufficiently long (and possibly infinite) prediction horizon for the zero state constraint case, or by choosing a robust stabilizing control in the dual mode case. We will also observe instances that violate this assumption for the case study presented in Section 5 .

\subsection{Perfect model case}

The essence of our stability analysis follows from familiar arguments summarized in [7]. We first consider the case where the model is perfect and there is no source of disturbances. From the assumptions stated in Section 2, the solu- tion of $\mathscr{P}_{\rho}\left(x_{i}\right)$ satisfies $\left(x_{k}, u_{k}\right)=(0,0)$ for $k \geqslant i+p$. Hence the locally optimal solution gives

$\Upsilon^{*}\left(x_{i}\right)=\sum_{k=i}^{i+p-1} h\left(x_{k}^{*}, u_{k}^{*}\right)+h_{\mathrm{F}}\left(x_{i+p}^{*}\right)+P\left(s_{i}^{*}, \rho\right)$.

Note that we assume the point $\left(x_{k}^{\mathrm{p}}, u_{k}\right)=\left(x_{k}, u_{k}\right)=(0,0)$ is within the state and control bound constraints.

Consider now the problem at the next time index, $\mathscr{P}_{\rho}\left(x_{i+1}\right)$. Because the model is perfect and there is no source of disturbances, the resulting optimal sequence of $\mathscr{P}_{\rho}\left(x_{i}\right)$ is a feasible solution for $\mathscr{P}_{\rho}\left(x_{i+1}\right)$. Moreover, the objective function at the solution of $\mathscr{P}_{\rho}\left(x_{i+1}\right)$ can be no greater than the solution $\mathscr{P}_{\rho}\left(x_{i}\right)$. Substituting $h_{\mathrm{F}}\left(x_{i+p}\right)=0$ for the zero state constraint case or applying (10) with $x=x_{i+p}$ for the dual mode case leads to the following relation:

$\Upsilon^{*}\left(x_{i}\right)-\Upsilon^{*}\left(x_{i+1}\right) \geqslant \tilde{h}\left(x_{i}, u_{i}^{*}, \rho\right)$,

where

$$
\begin{aligned}
& \tilde{h}\left(x_{i}, u_{i}^{*}, \rho\right)=h\left(x_{i}, u_{i}^{*}\right)+\rho \cdot \max \left\{0, b_{x}\left(x_{i}\right)\right\} \\
& \quad+\rho \cdot \max \left\{0, b_{u}\left(u_{i}^{*}\right)\right\} .
\end{aligned}
$$

Note also that $\tilde{h}\left(x_{i}, u_{i}^{*}, \rho\right) \in \mathscr{K}_{\infty}$. Taking the sum of (20) over $N$, noting that $\left\{\Upsilon^{*}\left(x_{i}\right)\right\}$ is nonincreasing and letting $N \rightarrow \infty$, leads to $\tilde{h}\left(x_{i}, u_{i}^{*}, \rho\right) \rightarrow 0$ and $x_{i} \rightarrow 0$.

\subsection{Model mismatch case}

Consider now the case with plant/model mismatch. To obtain the solution $\mathscr{P}_{\rho}(\cdot)$ at time index $i+1$ we have two initial state conditions available. One is the prediction made at time index $i$ for $i+1, \bar{x}_{i+1}$ from (19), and the other is defined by the state measurements at $i+1, x_{i+1}$ from (2). This leads to two MPC problems denoted by $\mathscr{P}_{\rho}\left(\bar{x}_{i+1}\right)$ and $\mathscr{P}_{\rho}\left(x_{i}+1\right)$, respectively. Both problems are solved with the same model (3), and the difference between their solutions reflects the degree of plant/model mismatch. From the perfect model case we assume that $\rho$ is large enough in order to obtain feasible solutions to $\mathscr{P}\left(\bar{x}_{i+1}\right)$ if they exist. However, since $x_{i+1}$ can be different from $\bar{x}_{i+1}$, we accept that the bound constraints may be violated and $P\left(s_{i+1}^{*}, \rho\right)>0$. Nevertheless, we will still assume that terminal constraints are satisfied for $x_{i+1}$.

To account for mismatch we add and subtract $\Upsilon^{*}\left(\bar{x}_{i+1}\right)$ to the difference $\Upsilon^{*}\left(x_{i}\right)-\Upsilon^{*}\left(x_{i+1}\right)$, and use (20) to get

$$
\begin{aligned}
\Upsilon^{*}\left(x_{i}\right)-\Upsilon^{*}\left(x_{i+1}\right)= & \Upsilon^{*}\left(x_{i}\right)-\Upsilon^{*}\left(\bar{x}_{i+1}\right)-\left(\Upsilon^{*}\left(x_{i+1}\right)\right. \\
& \left.-\Upsilon^{*}\left(\bar{x}_{i+1}\right)\right) \\
\geqslant & \tilde{h}\left(x_{i}, u_{i}^{*}, \rho\right)-\left(\Upsilon^{*}\left(x_{i+1}\right)-\Upsilon^{*}\left(\bar{x}_{i+1}\right)\right) \\
\geqslant & W\left(\left\|x_{i}\right\|\right),
\end{aligned}
$$

where the last inequality forces the right hand sides to be bounded by a positive function $W\left(\left\|x_{i}\right\|\right)$ of class $\mathscr{K}_{\infty}$. This ensures that asymptotic stability holds. Note that the difference $\Upsilon^{*}\left(x_{i+1}\right)-\Upsilon^{*}\left(\bar{x}_{i+1}\right)$ is a measure of the plant/model mismatch and will be referred to as the mismatch term. 


\section{The mismatch term}

To characterize the mismatch term we first invoke the mean value theorem to derive an expression for the mismatch term as a function of the difference between the two problem solutions. Then we consider the optimality conditions of both problems to derive a bound on the mismatch term, which leads to a sufficient condition for closedloop stability under the presence of plant/model mismatch.

\subsection{Preliminaries}

Assuming that a value of $\rho$ can be chosen that is sufficiently large, we slightly modify $\Upsilon^{*}(\cdot)$ by applying a smoothing function from Balakrishna and Biegler [1] to every element of (17), e.g., for a scalar $y$ :

$\max \left\{0, b_{x}(y)\right\} \approx b_{\xi x}(y, \xi)=\frac{b_{x}(y)+\sqrt{b_{x}(y)^{2}+\xi^{2}}}{2}$

with small $\xi>0$; the corresponding modification is also made for $b_{u}$. The definition (16) is now replaced by

$\Upsilon\left(x_{i}, \boldsymbol{s}_{i}, \rho_{i}, \xi\right)=\Psi\left(x_{i}, \boldsymbol{s}_{i}\right)+P_{\xi}\left(\boldsymbol{s}_{i}, \rho_{i}, \xi\right)$

with $P_{\xi}\left(\boldsymbol{s}_{i}, \rho_{i}, \xi\right)=\rho_{i}\left[\sum_{k=i+1}^{i+p-1} b_{\xi x}\left(x_{k}, \xi\right)+\sum_{k=i}^{i+m-1} b_{\xi u}\left(u_{k}, \xi\right)\right]$. $\Upsilon\left(x_{i}, s_{i}, \rho_{i}, \xi\right)$ is continuous and at least twice differentiable with respect to $s_{i}$. Invoking the mean value theorem yields

$$
\begin{aligned}
\Upsilon^{*}\left(x_{i+1}\right)-\Upsilon^{*}\left(\bar{x}_{i+1}\right)= & \int_{0}^{1}\left\{\frac{\mathrm{d}}{\mathrm{d} x}\left[\Upsilon^{*}\left(\bar{x}_{i+1}+\tau\left(x_{i+1}-\bar{x}_{i+1}\right)\right)\right]^{\mathrm{T}}\right\} \\
& \cdot\left(x_{i+1}-\bar{x}_{i+1}\right) \mathrm{d} \tau .
\end{aligned}
$$

From (21) and (23) we can write $\tilde{h}_{\xi}\left(x_{i}, u_{i}^{*}, \rho, \xi\right)=$ $h\left(x_{i}, u_{i}^{*}\right)+\rho \cdot b_{\xi x}\left(x_{i}, \xi\right)+\rho \cdot b_{\xi u}\left(u_{i}^{*}, \xi\right)$. Returning to the notation (18), defining

$\varepsilon_{i+k}^{*}=\left[s_{i+k}^{*}-\bar{s}_{i+k}^{*}\right]=\left[\begin{array}{l}x_{i+k}^{*}-\bar{x}_{i+k}^{*} \\ u_{i+k}^{*}-\bar{u}_{i+k}^{*}\end{array}\right]$

for $k=1, \ldots, p$, and substituting (6), (23) and (26), (25) into (24) leads to

$$
\begin{aligned}
\Upsilon^{*}\left(x_{i+1}\right)-\Upsilon^{*}\left(\bar{x}_{i+1}\right)= & \sum_{k=1}^{p} \int_{0}^{1} \nabla_{s_{i+k}} \tilde{h}_{\xi}\left(\bar{s}_{i+k}^{*}\right. \\
& \left.+\tau \varepsilon_{i+k}^{*}, \rho, \xi\right)^{\mathrm{T}} \varepsilon_{i+k}^{*} \mathrm{~d} \tau .
\end{aligned}
$$

Proceeding as in the unconstrained case study by Santos and Biegler [10] with $\Upsilon^{*}(\cdot)$ replacing $\Psi^{*}(\cdot)$ we derive the following bound on the mismatch term:

$$
\begin{aligned}
& \left|\Upsilon^{*}\left(x_{i+1}\right)-\Upsilon^{*}\left(\bar{x}_{i+1}\right)\right| \\
& \quad \leqslant \sum_{k=1}^{p}\left\|\int_{0}^{1} \nabla_{s_{i+k}} \tilde{h}_{\xi}\left(\bar{s}_{i+1}^{*}+\tau \varepsilon_{i+k}^{*}, \rho, \xi\right)^{\mathrm{T}} \mathrm{d} \tau\right\| \cdot \Gamma \cdot W_{\mathrm{m}}\left(\left\|x_{i}\right\|\right),
\end{aligned}
$$

where $\Gamma$ is a bound on variations of the stationarity conditions for $\mathscr{P}_{\rho}(\cdot)$ as defined by Eq. (4.12) in [10], with $\Upsilon^{*}(\cdot)$ replacing $\Psi^{*}(\cdot)$.
Moreover, we can consider more specific MPC objectives (6) defined by

$h\left(s_{i+k}\right)=s_{i+k}^{\mathrm{T}} Q_{i+k} s_{i+k}$,

where $Q_{i+k}=\operatorname{diag}\left\{Q_{x i+k}, Q_{u i+k}\right\}$, and $Q_{x i+k} \in \mathbb{R}^{n_{\mathrm{s}} \times n_{\mathrm{s}}}$ and $Q_{u i+k} \in \mathbb{R}^{n_{\mathrm{m}} \times n_{\mathrm{m}}}$ are diagonal matrices corresponding to the state and input weighting matrices at predictive horizon time index $i+k$, respectively. From (29), the analytical form of the integral term in (28) is (see Appendix A)

$$
\begin{aligned}
& \int_{0}^{1} \nabla_{s_{i+k}} \tilde{h}_{\xi}\left(\bar{s}_{i+k}^{*}+\tau \varepsilon_{i+k}^{*}, \rho, \xi\right)^{\mathrm{T}} \mathrm{d} \tau \\
& \quad=\left(2 \bar{s}_{i+k}^{*}+\varepsilon_{i+k}^{*}\right)^{\mathrm{T}} Q_{i+k}+\rho \cdot r\left(\bar{s}_{i+k}^{*}, \varepsilon_{i+k}^{*}, \xi\right),
\end{aligned}
$$

where $r\left(\bar{s}_{i+k}^{*}, \varepsilon_{i+k}^{*}, \xi\right)$ denotes a vector whose elements are nonlinear functions of $\bar{s}_{i+k}^{*}, \varepsilon_{i+k}^{*}$ and $\xi$. Following the same developments as in Santos and Biegler [10] we obtain

$$
\begin{aligned}
& \left|\Upsilon^{*}\left(x_{i+1}\right)-\Upsilon^{*}\left(\bar{x}_{i+1}\right)\right| \\
& \quad \leqslant \sum_{k=1}^{p}\left\{\left(\left\|2 \bar{s}_{i+k}^{*}\right\|+\left\|\varepsilon_{i+k}^{*}\right\|\right)\left\|Q_{i+k}\right\|\right. \\
& \left.\quad+\rho \cdot\left\|r\left(\bar{s}_{i+k}^{*}, \varepsilon_{i+k}^{*}, \xi\right)\right\|\right\} \cdot \Gamma \cdot W_{\mathrm{m}}\left(\left\|x_{i}\right\|\right) .
\end{aligned}
$$

We assume there are positive constants $Q, \alpha_{1}$ and $\alpha_{2}$, such that for all $i \geqslant 0$ and $k \leqslant p$

$\left\|r\left(\bar{s}_{i+k}^{*}, \varepsilon_{i+k}^{*}, \xi\right)\right\| \leqslant \alpha_{1}\left\|2 \bar{s}_{i+k}^{*}\right\|+\alpha_{2}\left\|\varepsilon_{i+k}^{*}\right\|$

and $\left\|Q_{i+k}\right\| \leqslant Q$. Moreover, since $\bar{s}_{i+k}^{*}, k=1, \ldots, p$, depends on $x_{i}$, we set

$\left\|2 \bar{s}_{i+k}^{*}\right\| \leqslant \widehat{K}\left\|x_{i}\right\|$,

where $\widehat{K}$ is a positive constant. From (5), with $\gamma=1$ (see $[10])$,

$\left\|\varepsilon_{i+k}^{*}\right\| \leqslant \Gamma \cdot W_{\mathrm{m}}\left(\left\|x_{i}\right\|\right) \leqslant \Gamma K_{\mathrm{m}}\left\|x_{i}\right\|$

for every $k, k \leqslant p$. Finally, substituting (32)-(34) in (31) leads to

$\left|\Upsilon^{*}\left(x_{i+1}\right)-\Upsilon^{*}\left(\bar{x}_{i+1}\right)\right| \leqslant K_{\mathrm{B}}\left\|x_{i}\right\|^{2}$,

where

$K_{\mathrm{B}}=p\left\{\left(\widehat{K}+\Gamma K_{\mathrm{m}}\right) Q+\rho \cdot\left(\alpha_{1} \widehat{K}+\alpha_{2} \Gamma K_{\mathrm{m}}\right)\right\} \Gamma K_{\mathrm{m}}$.

Note that the first term of the sum on the right hand side of (36) is the expression of $K_{\mathrm{B}}$ obtained for the unconstrained case [10]. Therefore, when there are no active constraints $\rho=0$ and (35) is equal to the unconstrained case sufficient stability bound. Also, from (22) and (35) it follows that

$$
\begin{aligned}
& \tilde{h}\left(x_{i}, u_{i}^{*}, \rho\right)-\left|\Upsilon^{*}\left(x_{i+1}\right)-\Upsilon^{*}\left(\bar{x}_{i+1}\right)\right| \\
& \quad \geqslant h\left(x_{i}, u_{i}^{*}\right)-K_{\mathrm{B}}\left\|x_{i}\right\|^{2}=W\left(\left\|x_{i}\right\|\right) .
\end{aligned}
$$

In particular, consider the case where $Q_{x i}=\alpha_{x} I$ and $Q_{u i}=\alpha_{u} I$, with constants $\alpha_{x}>0$ and $\alpha_{u} \geqslant 0$. From solution of $\mathscr{P}\left(x_{i}\right) u_{i}^{*}$ is an implicit function of $x_{i}$, so we can write 
$\left\|u_{i}^{*}\right\|^{2}=\beta\left\|x_{i}\right\|^{2}$, for some $\beta>0$. Thus, without constraint violations,

$h\left(x_{i}, u_{i}^{*}\right)=\alpha_{x} x_{i}^{\mathrm{T}} x_{i}+\alpha_{u} u_{i}^{* \mathrm{~T}} u_{i}^{*}=\left(\alpha_{x}+\alpha_{u} \beta\right)\left\|x_{i}\right\|^{2}$.

For a given $x_{i}$, and with $\alpha_{u}=0$, it follows from (37) that $K_{\mathrm{B}}<\alpha_{x}$ satisfies the sufficient condition for stability. When $\alpha_{u} \neq 0$, this condition is relaxed to

$K_{\mathrm{B}}<\alpha_{x}+\alpha_{u} \beta$.

\subsection{A tool to analyze robust stability}

Because $\beta$ in (39) depends on the optimization problem solution it is difficult to calculate $K_{\mathrm{B}}$ a priori. Moreover, when constraint violations occur a tighter value of the sufficient stability condition for the constrained case, $K_{\mathrm{B}}$, can be estimated by exploiting the state-space region of interest from

$K_{\mathrm{B}} \geqslant \max _{x_{i}} \frac{\left|\Upsilon^{*}\left(x_{i+1}\right)-\Upsilon^{*}\left(\bar{x}_{i+1}\right)\right|}{\left\|x_{i}\right\|^{2}}$.

This procedure involves the off-line calculation of $K_{\mathrm{B}}$ according to the following cycle:

1. For a given $x_{i}, i \geqslant 0$, perform the following steps:

2. Solve $\mathscr{P}_{\rho}\left(x_{i}\right)$; save $\bar{x}_{i+1}$.

3. Implement $u_{i}^{*}$ and set $i=i+1$.

(i) Using $x_{i+1}$, solve $\mathscr{P}_{\rho}\left(x_{i+1}\right)$ to obtain $\Upsilon^{*}\left(x_{i+1}\right)$.

(ii) Using $\bar{x}_{i+1}$, solve $\mathscr{P}_{\rho}\left(\bar{x}_{i+1}\right)$ to obtain $\Upsilon^{*}\left(\bar{x}_{i+1}\right)$.

(iii) Go to 1 and repeat steps with new values of $x_{i}$.

4. Set

$$
K_{\mathrm{B}}=\max _{i} \frac{\left|\Upsilon^{*}\left(x_{i+1}\right)-\Upsilon^{*}\left(\bar{x}_{i+1}\right)\right|}{\left\|x_{i}\right\|^{2}}+\eta,
$$

where $\eta>0$ is a user-specified safety factor. Therefore for a nonzero $x_{i}$ we can compute a reasonable approximation to $K_{\mathrm{B}}$.

\section{Illustrative example}

To demonstrate the above approach, we consider an exothermic zero-order reaction system, $\mathrm{A} \rightarrow \mathrm{B}$, with concentration and temperature dynamics described by

$$
\begin{aligned}
\frac{\mathrm{d} C_{\mathrm{A}}}{\mathrm{d} t} & =\frac{F_{0}}{V}\left(C_{\mathrm{A}_{0}}-C_{\mathrm{A}}\right)-k_{0} \mathrm{e}^{-E_{\mathrm{a}} /\left(R T_{\mathrm{r}}\right)}, \\
\frac{\mathrm{d} T_{\mathrm{r}}}{\mathrm{d} t} & =\frac{1}{\rho_{\mathrm{L}} C_{\mathrm{p}} V}\left(-Q_{\mathrm{R}}+Q_{\mathrm{G}}\right),
\end{aligned}
$$

where $Q_{\mathrm{R}}$ is the rate of heat removal and $Q_{\mathrm{G}}$ is the rate of heat generated by the reaction, given by

$Q_{\mathrm{R}}=-\rho C_{\mathrm{p}} F_{0}\left(T_{0}-T_{\mathrm{r}}\right)+U A\left(T_{\mathrm{r}}-T_{\mathrm{j}}\right)$,

$Q_{\mathrm{G}}=\left(-\Delta H_{\mathrm{r}}\right) V k_{0} \mathrm{e}^{-E_{\mathrm{a}} /\left(R T_{\mathrm{r}}\right)}$.

The steady state operating conditions - except for $C_{\mathrm{A}}$ whose value is an initial condition - and parameter values for this case study are given in Table 1 .
Table 1

Steady state operating conditions and model parameters

\begin{tabular}{lll}
\hline$A$ & Heat transfer area & $0.623 \mathrm{~m}^{2}$ \\
$C_{\mathrm{A}}, C_{\mathrm{A} 0}$ & $\begin{array}{l}\text { Reactor and inlet } \\
\text { concentrations of A }\end{array}$ & $10.0 \mathrm{~mol} / 1$ \\
$C_{\mathrm{p}}$ & Heat capacity of the liquid & $4184 \mathrm{~J} / \mathrm{kg} \mathrm{K}$ \\
$E_{\mathrm{a}} / R$ & Activation energy over $R$ & $10,080 \mathrm{~K}$ \\
$F_{0}$ & Feed flow rate & $4.001 / \mathrm{min}$ \\
$k_{0}$ & Arrhenius constant & $6.20 \times 10^{14} \mathrm{~mol} / \mathrm{m}^{3} \mathrm{~s}$ \\
$\bar{T}_{\mathrm{j}}, \bar{T}_{\mathrm{r}}$ & Coolant and reactor temperatures & $34.0^{\circ} \mathrm{C}$ \\
$\bar{T}_{0}$ & Feed temperature & $11.1^{\circ} \mathrm{C}$ \\
$U$ & Overall heat transfer coef. & $V$ liquid volume $0.0551 \mathrm{~m}^{3}$ \\
& $300 \mathrm{~W} / \mathrm{m}^{2} \mathrm{~K}$ & \\
$\left(-\Delta H_{\mathrm{r}}\right)$ & Heat of reaction, 33,488 J/mol & $\rho_{\mathrm{L}}$ liquid density, \\
& & $1000 \mathrm{~kg} / \mathrm{m}^{3}$ \\
\hline
\end{tabular}

Since the reaction is zero order the evolution of $T_{\mathrm{r}}$ is independent of the evolution of $C_{\mathrm{A}}$ and also (45) does not depend on $C_{\mathrm{A}}$. This nonlinear system is open loop unstable under certain operating conditions. Fig. 1 shows the reactor temperature open loop response for different initial reactor temperature conditions $-T_{\mathrm{r}, i}=33,34$ and $35^{\circ} \mathrm{C}$. Clearly, for $T_{\mathrm{r}, i}>34^{\circ} \mathrm{C}$, the system is open loop unstable. A more detailed description of this system can be found in Santos [9].

\subsection{Testing for the sufficient stability condition}

The control objective is to control $T_{\mathrm{r}}$ - the set-point is $T_{\mathrm{r} \mathrm{sp}}=34{ }^{\circ} \mathrm{C}-$ by manipulating the cooling fluid temperature $T_{\mathrm{j}}$ subject to the operating constraints $T_{\mathrm{r}} \geqslant 0{ }^{\circ} \mathrm{C}$ and $T_{\mathrm{j}} \geqslant 15^{\circ} \mathrm{C}$. To satisfy these constraints the control problems are solved using (16) with $\rho=1000$. We set $\left(\alpha_{x}, \alpha_{u}\right)=(1,0)$, predictive horizons $(p, m)=(25,1), \Delta t=$ $0.5 \mathrm{~min}$ and we impose a zero state terminal constraint. The steady state data $\left(\bar{T}_{\mathrm{j}}, \bar{T}_{\mathrm{r}}\right.$ and $\left.\bar{T}_{0}\right)$ in Table 1 satisfy the assumption in Section 2 that - using deviation variables $-\left(x_{k}, u_{k}\right)=(0,0)$ is the point at which both the plant and the model operate at steady state. We emphasize that this assumption is satisfied for all the cases of plant/model mismatch discussed below. To test for the sufficient stability condition, we consider parametric model mismatch on the overall heat transfer coefficient $U$. Fig. 2 shows the variation of $K_{\mathrm{B}}$ with $T_{\mathrm{r}, i}$ varying in the operating region of interest, from 24 to $44{ }^{\circ} \mathrm{C}$, and for various model mismatch values, $U_{\mathrm{m}}=200,300,400$ and $500 \mathrm{~W} / \mathrm{m}^{2} \mathrm{~K}$. The true plant value is $U_{\mathrm{p}}=300 \mathrm{~W} / \mathrm{m}^{2} \mathrm{~K}$. Each dot in Fig. 2 corresponds to one cycle of the procedure to calculate $K_{\mathrm{B}}$ (Section 4.2). Thus, we observe that in the case of perfect model $\left(U_{\mathrm{m}}=300 \mathrm{~W} / \mathrm{m}^{2} \mathrm{~K}\right), K_{\mathrm{B}}=0$.

We also emphasize that when the terminal constraint is not satisfied our assumption from Section 3 fails, a finite value of $K_{\mathrm{B}}$ cannot be calculated and the system is obviously not robustly stable. This case is illustrated in Fig. 2 by the vertical dashed lines in the region $T_{\mathrm{r}, i}>34{ }^{\circ} \mathrm{C}$. Here, the nonexistence of a feasible solution can be overcome by increasing the prediction horizon $p$ appropriately to allow the calculation of $K_{\mathrm{B}}$. 

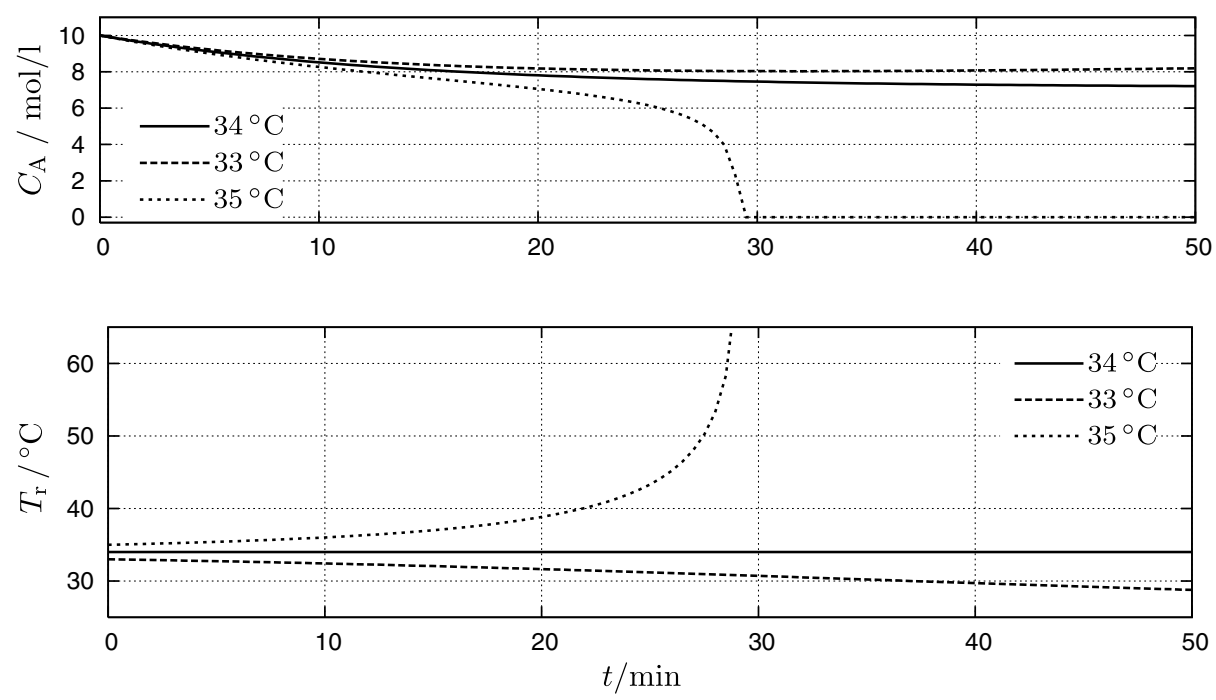

Fig. 1. Open loop response to different initial state conditions, $T_{\mathrm{r}, i}$.

As discussed in Section 4.2, when the state and input weighting matrices are set such that $\left(\alpha_{x}, \alpha_{u}\right)=(1,0)$, a sufficient condition for robust stability from (37) requires
$K_{\mathrm{B}}<1$. This can be seen in Figs. 2 and 3. Based on the performance of the MPC controller, calculation of $K_{\mathrm{B}}$ can separated into two domains for $T_{\mathrm{r}, i}$. For $T_{\mathrm{r}, i}<34^{\circ} \mathrm{C}$, the

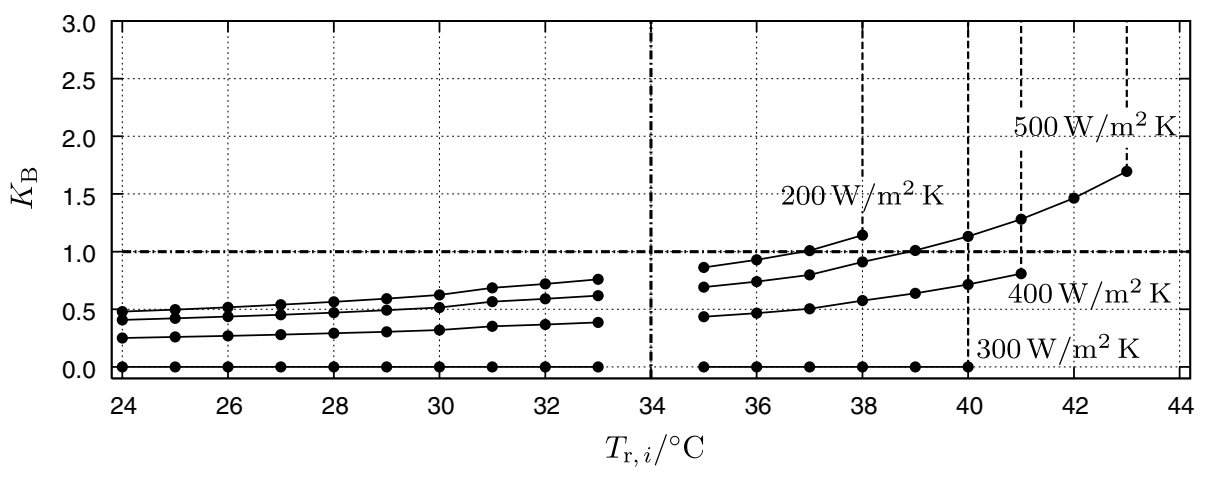

Fig. 2. Variation of the constant bound.
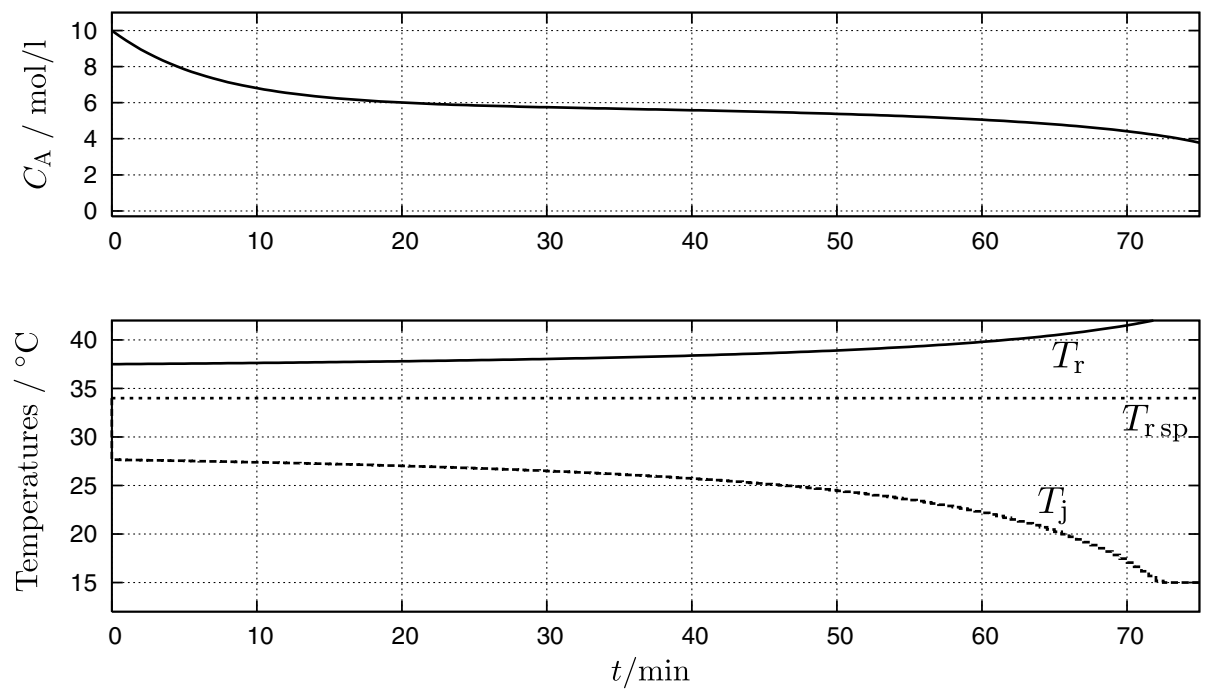

Fig. 3. Unstable regulator control response. 
profiles always show $K_{\mathrm{B}}<1$. Under these conditions the system is robustly stable and all of our simulated cases converge to the origin (set-point) of $T_{\mathrm{r}}=34^{\circ} \mathrm{C}$.

For $T_{\mathrm{r}, i}>34^{\circ} \mathrm{C}$, the $K_{\mathrm{B}}$ profiles cross the line $K_{\mathrm{B}}=1$ as $T_{\mathrm{r}, i}$ increases. For example, with $U_{\mathrm{m}}=500 \mathrm{~W} / \mathrm{m}^{2} \mathrm{~K}$, the system is unstable, as $K_{\mathrm{B}}>1$ for $T_{\mathrm{r}, i} \geqslant 39^{\circ} \mathrm{C}$. For this case, the MPC controller does not converge for initial values of $T_{\mathrm{r}, i}>34{ }^{\circ} \mathrm{C}$. Moreover, with $U_{\mathrm{m}}=400 \mathrm{~W} / \mathrm{m}^{2} \mathrm{~K}$, the system is also unstable because the terminal constraint cannot be satisfied in this region, i.e. for $T_{\mathrm{r}, i}=41^{\circ} \mathrm{C}$. Performance of this case can be seen in Fig. 3 with $T_{\mathrm{r}, i}=37.5^{\circ} \mathrm{C}$ and $U_{\mathrm{m}}=400 \mathrm{~W} / \mathrm{m}^{2} \mathrm{~K}$. After $t \approx 72 \mathrm{~min}$ the manipulated variable is set by the controller at its lower limit. Note that for both $U_{\mathrm{m}}=400$ and $500 \mathrm{~W} / \mathrm{m}^{2} \mathrm{~K}$ the cooling rate calculated by the controller is not sufficient to cool down the reactor liquid and therefore a temperature runaway occurs.

On the other hand, because the robust stability condition is based on normed quantities, it is only sufficient. Stable performance may still be possible because calculation of $K_{\mathrm{B}}$ is based on magnitudes and not the sign of the mismatch terms in (37) and (40). For instance, for $U_{\mathrm{m}}=200 \mathrm{~W} / \mathrm{m}^{2} K$ we also have $K_{\mathrm{B}}>1$ when $T_{\mathrm{r}, i} \geqslant 37^{\circ} \mathrm{C}$. However, for $U_{\mathrm{p}}>U_{\mathrm{m}}$, the control solution is actually favorable to the plant because the controller provides a greater cooling rate than really necessary, and thus has a stronger dampening effect.

\section{Conclusions}

We develop a strategy based on nonlinear programming sensitivity that determines conditions under which the constrained model predictive control is robustly stable with respect to modeling errors. The approach applies to finite horizon MPC formulations using either zero state terminal constraints or the dual mode formulation. Here, a sufficient condition for robust stability is derived and an off-line procedure is developed to evaluate constants which determine sufficient conditions for this property. These constants are available from bounds on the model mismatch and from the NLP solution of the receding horizon model. This procedure is applicable to both linear and nonlinear model predictive controllers in discrete time that satisfy nominal stability properties based on Lyapunov arguments.

\section{Appendix A. Quadratic function and finite horizon}

To obtain the analytical form of the integral term in (28) we recall the smoothing function (23) applied to every element of (17). From (8) let us define, for a scalar $y$, $b_{\mathrm{U}}(y)=y-y_{\mathrm{U}}$ and $b_{\mathrm{L}}(y)=-y+y_{\mathrm{L}}$. Therefore

$$
\begin{aligned}
& \tilde{h}_{\xi}(y, \rho, \xi)=y^{2} q_{y}+\rho \cdot\left(b_{\mathrm{U} \xi y}(y, \xi)+b_{\mathrm{L} \xi y}(y, \xi)\right)=y^{2} q_{y} \\
& \quad+\frac{\rho}{2} \cdot\left[\sqrt{b_{\mathrm{U}}(y)^{2}+\xi^{2}}+b_{\mathrm{U}}(y)+\sqrt{b_{\mathrm{L}}(y)^{2}+\xi^{2}}+b_{\mathrm{L}}(y)\right]
\end{aligned}
$$

with constant $q_{y} \geqslant 0$. Taking the derivative of $\tilde{h}_{\xi}(y, \rho, \xi)$ with respect to $y$ leads to

$$
\frac{\mathrm{d}}{\mathrm{d} y} \tilde{h}_{\xi}(y, \rho, \xi)=2 y q_{y}+\frac{\rho}{2} \cdot\left[\frac{b_{\mathrm{U}}(y)}{\sqrt{b_{\mathrm{U}}(y)^{2}+\xi^{2}}}-\frac{b_{\mathrm{L}}(y)}{\sqrt{b_{\mathrm{L}}(y)^{2}+\xi^{2}}}\right] .
$$

Finally, replacing $y$ by $\bar{y}+\tau \varepsilon$ and integrating between 0 and 1 gives

$$
\begin{aligned}
\int_{0}^{1} & \frac{\mathrm{d}}{\mathrm{d} y} \tilde{h}_{\xi}(\bar{y}+\tau \varepsilon, \rho, \xi) \mathrm{d} \tau \\
= & 2 q_{y}\left(\bar{y}+\frac{\varepsilon}{2}\right)+\frac{\rho}{2 \varepsilon} \cdot\left(\sqrt{\left(\bar{y}+\varepsilon-y_{\mathrm{U}}\right)^{2}+\xi^{2}}\right. \\
& -\sqrt{\left(\bar{y}-y_{\mathrm{U}}\right)^{2}+\xi^{2}}+\sqrt{\left(-\bar{y}-\varepsilon+y_{\mathrm{L}}\right)^{2}+\xi^{2}} \\
& \left.-\sqrt{\left(-\bar{y}+y_{\mathrm{L}}\right)^{2}+\xi^{2}}\right) \\
= & 2 q_{y}\left(\bar{y}+\frac{\varepsilon}{2}\right)+\frac{\rho}{2 \varepsilon} \cdot\left(\sqrt{\left(b_{\mathrm{U}}(\bar{y})+\varepsilon\right)^{2}+\xi^{2}}\right. \\
& -\sqrt{b_{\mathrm{U}}(\bar{y})^{2}+\xi^{2}}+\sqrt{\left(b_{\mathrm{L}}(\bar{y})-\varepsilon\right)^{2}+\xi^{2}} \\
& \left.-\sqrt{b_{\mathrm{L}}(\bar{y})^{2}+\xi^{2}}\right)=2 q_{y}\left(\bar{y}+\frac{\varepsilon}{2}\right)+\rho \cdot r(\bar{y}, \varepsilon, \xi) .
\end{aligned}
$$

From the comparison with the unconstrained case [10] this analytical form has an additional term which is a nonlinear function of $\bar{y}, \varepsilon$ and $\xi$, denoted by $r(\bar{y}, \varepsilon, \xi)$. It is straightforward to obtain (30) from (A.1) using the array definitions in (26).

\section{References}

[1] S. Balakrishna, L.T. Biegler, Targeting strategies for the synthesis and energy integration of nonisothermal reactor networks, Industrial \& Engineering Chemistry Research 31 (1992) 2152-2164.

[2] R. Fletcher, Practical Methods of Optimization, second ed., John Wiley \& Sons Ltd., Chichester, 1987.

[3] S.S. Keerthi, E.G. Gilbert, Optimal infinite-horizon feedback laws for general class of constrained discrete-time systems: stability and moving-horizon approximations, Journal of Optimization Theory and Applications 57 (2) (1988) 265-293.

[4] L. Magni, R. Scattolini, Control design for nonlinear systems: tading robustness and performance with the model predictive control approach, IEE Proceedings - Control Theory \& Application 152 (3) (2005) 333-339.

[5] L. Magni, R. Scattolini, Robustness and robust design of MPC for nonlinear discrete-time systems, in: F. Allgöwer, R. Findeisen, L. Biegler (Eds.), Assessment and Future Directions of Nonlinear Model Predictive Control, Lecture Notes in Control and Information Sciences, vol. 358, Springer-Verlag, 2007, pp. 239-254.

[6] D.L. Marruedo, T. Alamo, E.F. Camacho, Input-to-state stable MPC for constrained discrete-time nonlinear systems with bounded additive uncertainties, IEEE CDC (2002) 4619-4624.

[7] D.Q. Mayne, J.B. Rawlings, C.V. Rao, P.O.M. Scokaert, Constrained model predictive control: optimality and stability, Automatica 36 (6) (2000) 789-814.

[8] N.M.C. Oliveira, L.T. Biegler, Constraint handling and stability properties of model predictive control, AIChE Journal 40 (7) (1994) $1138-1155$. 
[9] L.O. Santos, Multivariable Predictive Control of Chemical Processes, $\mathrm{Ph} . \mathrm{D}$. thesis, Faculty of Science and Technology, University of Coimbra, Coimbra, Portugal, 2001.

[10] L.O. Santos, L.T. Biegler, A tool to analyze robust stability for model predictive controllers, Journal of Process Control 9 (1999) 233-246.
[11] L.O. Santos, N.M.C. Oliveira, L.T. Biegler, Reliable and efficient optimization strategies for nonlinear model predictive control, in: J.B. Rawlings (Ed.), Proceedings of DYCORD+'95, Helsingør, Denmark, Elsevier Science, Oxford, 1995 